ARTICLE

https://doi.org/10.1038/s41467-019-11796-4

\title{
Directly transforming copper (I) oxide bulk into isolated single-atom copper sites catalyst through gas-transport approach
}

\author{
Zhengkun Yang ${ }^{1,7}$, Bingxu Chen ${ }^{2,7}$, Wenxing Chen ${ }^{3,7}$, Yunteng Qu ${ }^{1}$, Fangyao Zhou ${ }^{1}$, Changming Zhao ${ }^{1}$, Qian Xu ${ }^{4}$, \\ Qinghua Zhang ${ }^{5}$, Xuezhi Duan ${ }^{2}$ \& Yuen Wu (i) ${ }^{1,6}$
}

Single-atom metal catalysts have sparked tremendous attention, but direct transformation of cheap and easily obtainable bulk metal oxide into single atoms is still a great challenge. Here we report a facile and versatile gas-transport strategy to synthesize isolated single-atom copper sites (Cu ISAS/NC) catalyst at gram levels. Commercial copper (I) oxide powder is sublimated as mobile vapor at nearly melting temperature $(1500 \mathrm{~K})$ and subsequently can be trapped and reduced by the defect-rich nitrogen-doped carbon (NC), forming the isolated copper sites catalyst. Strikingly, this thermally stable Cu ISAS/NC, which is obtained above $1270 \mathrm{~K}$, delivers excellent oxygen reduction performance possessing a recorded half-wave potential of $0.92 \mathrm{~V}$ vs RHE among other Cu-based electrocatalysts. By varying metal oxide precursors, we demonstrate the universal synthesis of different metal single atoms anchored on NC materials (M ISAS/NC, where M refers to Mo and Sn). This strategy is readily scalable and the as-prepared sintering-resistant M ISAS/NC catalysts hold great potential in high-temperature applications.

\footnotetext{
${ }^{1}$ School of Chemistry and Materials Science, iChEM, Hefei National Laboratory for Physical Sciences at the Microscale, University of Science and Technology of China, 230026 Hefei, China. ${ }^{2}$ State Key Laboratory of Chemical Engineering, East China University of Science and Technology, 130 Meilong Road, 200237 Shanghai, China. ${ }^{3}$ Beijing Key Laboratory of Construction Tailorable Advanced Functional Materials and Green Applications, School of Materials Science and Engineering, Beijing Institute of Technology, 100081 Beijing, PR China. ${ }^{4}$ National Synchrotron Radiation Laboratory (NSRL), 230026 Hefei, China. ${ }^{5}$ China Institute of Physics, Chinese Academy of Sciences, 100190 Beijing, China. ${ }^{6}$ Fujian Institute of Innovation, Chinese Academy of Sciences, Beijing, China. ${ }^{7}$ These authors contributed equally: Zhengkun Yang, Bingxu Chen, Wenxing Chen. Correspondence and requests for materials should be addressed to X.D. (email: xzduan@ecust.edu.cn) or to Y.W. (email: yuenwu@ustc.edu.cn)
} 
solated single-atom sites catalysts (ISASC) have attracted a great deal of research interests, because of their superior activity and selectivity for many chemical reactions ${ }^{1-3}$. A variety of synthetic methods, including physical and chemical routes, have been developed to fabricate ISASC in recent years. The physical approaches, such as atomic layer deposition $(\mathrm{ALD})^{4,5}$, mass-selected soft-landing technique ${ }^{6}$, face the drawbacks of low yields, complicated equipments and high costs, hindering their wide applications. The traditional chemical routes, such as wet impregnation ${ }^{7,8}$, coprecipitation, and photodeposition $^{9-11}$, usually involve tedious synthetic steps, including adsorption and further reduction of metal precursors and stabilization on defect-rich supports. Moreover, due to the lack of strong interaction between single atoms and supports, aggregation of single atoms into clusters or nanoparticles is inevitable to some extent under a real catalytic condition, especially the high reaction temperature, thus hampering the practical industrial applications of ISASC. Therefore, to satisfy the industrial requirements such as large-scale production and excellent repeatability, developing advanced synthetic methodology is urgently required but remains challenge for the preparation of ISASC.

Recently, the direct conversion from nanoparticles to ISASC is regarded as a promising strategy since a pioneering work reported by Datye and co-workers, in which platinum single atoms anchored on $\mathrm{CeO}_{2}$ nanorods were constructed by thermal diffusion from platinum nanoparticles $(\mathrm{NPs})^{12}$. Subsequently, Wei et al. ${ }^{13}$ also reported the direct transformation of noble metal NPs-to-single atom by in situ environmental transmission electron microscopy. Yang et al. ${ }^{14}$ described that the Ni NPs distributed on the surface of defect-containing $\mathrm{N}$-doped carbon can be converted into surface-bound single $\mathrm{Ni}$ atoms. But studies of the direct fabrication of ISASC from cheap and readily available bulk metal materials have rarely been reported. Recently, our group demonstrated an atoms emitting and trapping strategy that can transform bulk transition metals ( $\mathrm{Cu}, \mathrm{Co}$, and $\mathrm{Ni}$ foils) to metal ISASC with assistance of $\mathrm{NH}_{3}{ }^{15}$. However, metals generally exist in the form of oxide minerals under natural condition. Meanwhile, the utilization of corrosive $\mathrm{NH}_{3}$ will lead to harsh experiments and increase its cost to synthesize ISASC. Therefore, the direct construction of ISASC from cost-effective and commercial bulk metal oxide under non-corrosive gas protection is a more convenient route but not been achieved.

Herein, we demonstrate a high-temperature gas-transport strategy to directly transform a series of commercial available metal oxides into isolated single atoms onto the nitrogen-doped carbon (NC) with ease of mass-production. As shown in Fig. 1a, commercial copper (I) oxide $\left(\mathrm{Cu}_{2} \mathrm{O}\right)$ power and $\mathrm{NC}$ are a
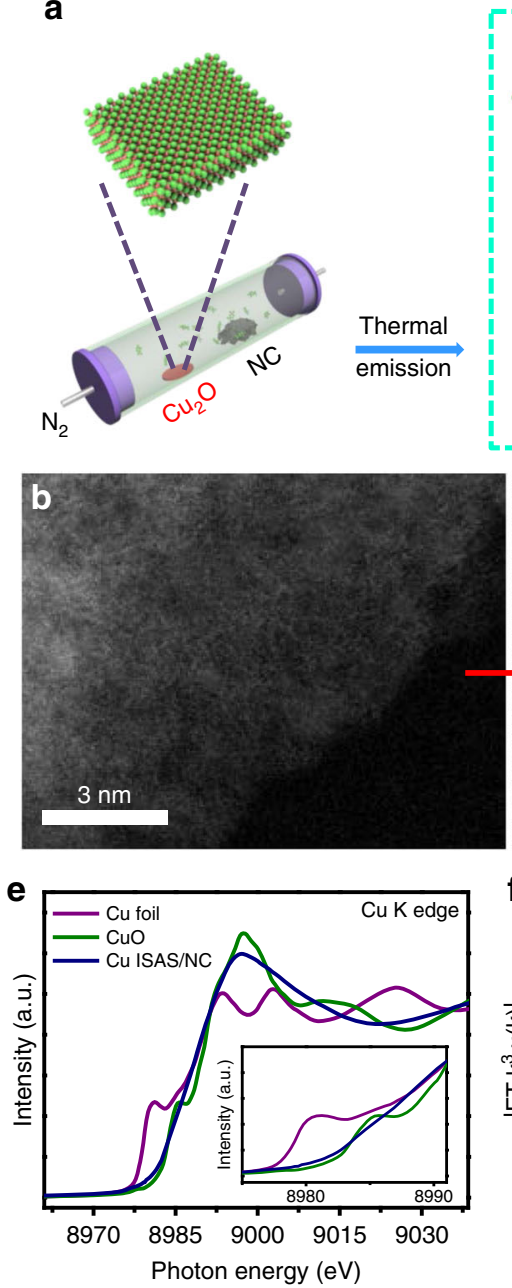
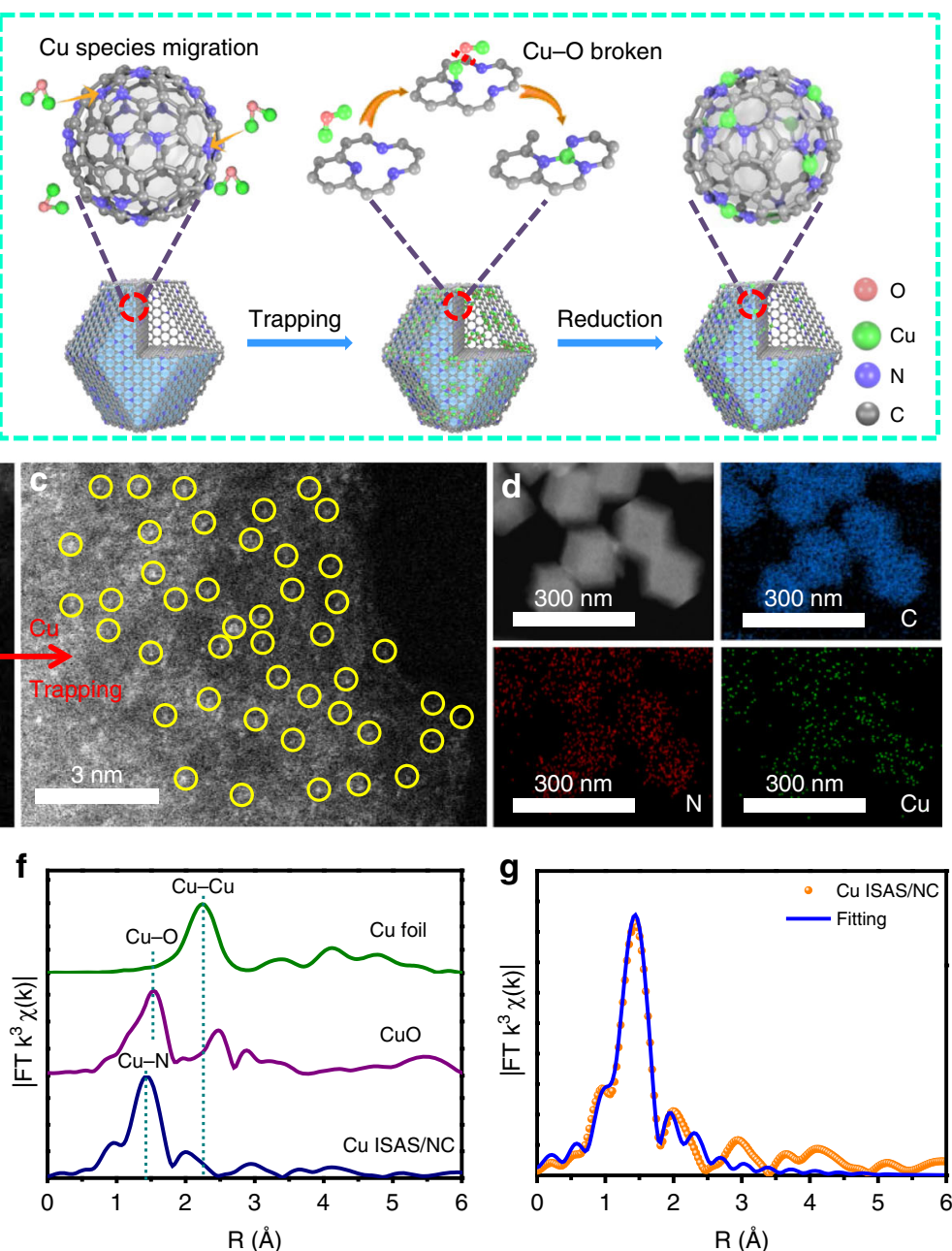

Fig. 1 Synthesis and structural characterizations. a Scheme of the formation isolated copper sites (Cu ISAS/N-C) catalyst. b aberration-corrected high-angle annular dark-field scanning transmission electron microscope (AC HAADF-STEM) image of NC. c AC HAADF-STEM image of Cu ISAS/NC. d Corresponding EDS mapping of Cu ISAS/NC. e Cu K-edge X-ray absorption near-edge structure (XANES) and $\mathbf{f}$ FT $\mathrm{k}^{3}$-weighted extended X-ray absorption fine structure (EXAFS) spectra of $\mathrm{Cu}$ ISAS/NC and the reference samples. $\mathbf{g}$ Corresponding FT-EXAFS fitting curves of Cu ISAS/NC 
separately located in the porcelain boat. At $1273 \mathrm{~K}$ in flowing $\mathrm{N}_{2}$, the surface $\mathrm{Cu}_{2} \mathrm{O}$ is initially evaporated to form volatile species, which can be trapped and reduced by the N-riched carbon support, forming the isolated $\mathrm{Cu}$ ISAS/NC catalyst. The volatility of $\mathrm{Cu}_{2} \mathrm{O}$ leads to the avoidance of corrosive $\mathrm{NH}_{3}$, benefiting for the large-scale production and practical applications. Importantly, a series of $\mathrm{M}$ ISAS/NC $(\mathrm{M}=\mathrm{Mo}, \mathrm{Sn})$ can be fabricated by changing metal oxide precursors, demonstrating its generality to construct a variety of functional ISASC.

\section{Results}

Synthesis and characterization of $\mathrm{Cu}$ ISAS/NC. The NC matrix with abundant $\mathrm{N}$-rich vacancies was prepared by a simple pyrolysis of ZIF-8 metal-organic frameworks (Supplementary Figs. $1-3)$ by selectively removing the volatile $\mathrm{Zn}$ nodes. As shown in Fig. 1b, aberration-corrected high-angle annular dark-field scanning transmission electron microscope (AC HAADF-STEM) image exhibits that there is no observable heavier metal atoms on the NC substrate. Supplementary Figure 4 shows that the $\mathrm{Cu}$ ISAS/NC retains the initial rhombododecahedral morphology and displays homogeneous size distribution, indicative of the trapping of $\mathrm{Cu}$ atoms would not result in the surface distortion and architecture collapsion. The AC HAADF-STEM images (Fig. 1c and Supplementary Fig. 5) present high-density of individual bright dots, validating the $\mathrm{Cu}$ species are atomically dispersed on the support. The energy-dispersive X-ray spectroscopy (EDS) analysis reveals the uniform distribution of $\mathrm{Cu}, \mathrm{C}$ and $\mathrm{N}$ (Fig. 1d). The actual content of $\mathrm{Cu}$ is about $0.45 \%$ measured by inductively coupled plasma atomic emission spectroscopy (ICP-AES) analysis. X-ray diffraction (XRD) pattern of Cu ISAS/ NC displays a broad peaks at $26^{\circ}$ (Supplementary Fig. 6a), which is related to the (002) plane of graphitic carbon ${ }^{16,17}$. No peaks of impurities such as $\mathrm{Cu}$ and $\mathrm{CuO}$ crystals were detected, in accordance with Raman spectrum (Supplementary Fig. 6b). X-ray photoelectron spectroscopy (XPS) spectrum of $\mathrm{Cu}$ ISAS/NC indicates the existence of $\mathrm{C}, \mathrm{N}$ and $\mathrm{O}$ and $\mathrm{Cu}$ (Supplementary Fig. 7). The high-resolution N1s (Supplementary Fig. 8a) can be deconvoluted into pyridinic-N $(398.4 \mathrm{eV})$, pyrrolic-N $(399.9 \mathrm{eV})$ and graphitic-N $(400.8 \mathrm{eV})^{18-20}$. From the high-resolution $\mathrm{C} 1 \mathrm{~s}$ spectrum (Supplementary Fig. 8b), three peaks are ascribed to graphitic $\mathrm{sp}^{2}$ carbon and nitrogen-bonded carbon ${ }^{21}$.

Atomic structure analysis of $\mathrm{Cu}$ ISAS/N-C by XAFS. To gain the chemical state and coordination environment of $\mathrm{Cu}$ ISAS/NC in atomic insight, X-ray absorption near-edge structure (XANES) and extended X-ray absorption fine structure (EXAFS) were conducted. The $\mathrm{Cu}$ K-edge XANES profiles in Fig. 1e suggest the oxidation valence state of the isolated single $\mathrm{Cu}$ atoms in $\mathrm{Cu}$ ISAS/NC is likely to be higher than metallic $\mathrm{Cu}^{0}$ and lower than $\mathrm{Cu}^{2+}$. The FTEXAFS curve of $\mathrm{Cu}$ ISAS/NC sample (Fig. 1f) shows the main peak at approximately $1.5 \AA$, which is attributed to the scattering interaction between the $\mathrm{Cu}$ atoms and the first shell $(\mathrm{Cu}-\mathrm{N})^{22}$. The WT plot of $\mathrm{Cu}$ ISAS/NC (Supplementary Fig. 9) just displays the intensity maximum at $5 \AA^{-1}$, assigning to the $\mathrm{Cu}-\mathrm{N}$ coordination. The local atomic structure around $\mathrm{Cu}$ in $\mathrm{Cu}$ ISAS/NC by EXAFS fitting matches well with the $\mathrm{Cu}-\mathrm{N}_{3}$ model (Fig. 1g, Supplementary Fig. 10 and Supplementary Table 1). The room temperature electron paramagnetic resonance (EPR) reveals the coordinatively unsaturated state of $\mathrm{Cu}$ species (Supplementary Fig. 11), demonstrating the existence of carbon vacancies. The $\mathrm{N} \mathrm{K}$-edge near-edge X-ray absorption fine structure (NEXAFS) spectrum shows three obvious peaks (Supplementary Fig. 12a), which result from $\pi^{*}$ transition in the C-N-C portion of the pyridinic- $\mathrm{N}$ site $(399.5 \mathrm{eV})$ and $\mathrm{N}-3 \mathrm{C}$ bridging of the graphitic- $\mathrm{N}$ site $(402.4 \mathrm{eV})$, and $\sigma^{*}$ transition of the $\mathrm{C}-\mathrm{N}$ bond $(408.5 \mathrm{eV})^{23,24}$. For $\mathrm{C}$ K-edge NEXAFS spectrum shown in Supplementary Fig. 12b, the peak A $(285.6 \mathrm{eV})$ and peak $\mathrm{B}(285.6 \mathrm{eV})$ derive from $\pi^{*}$ excitations of $\mathrm{C}=\mathrm{C}$ (ring) and $\mathrm{C}-\mathrm{N}-\mathrm{C}$, respectively, and the peak $\mathrm{C}(293.2 \mathrm{eV})$ originates from $\mathrm{C}-\mathrm{C} \sigma^{\star}$ (ring) transition ${ }^{25,26}$.

Electrocatalytic ORR performance of $\mathrm{Cu}$ ISAS/NC. Nitrogen $\left(\mathrm{N}_{2}\right)$ adsorption/desorption isotherms demonstrate that $\mathrm{Cu}$ ISAS/ NC has a high Brunauer-Emmett-Teller (BET) surface area of $831 \mathrm{~m}^{2} \mathrm{~g}^{-1}$ (Supplementary Fig. 13), due to their highly porous structure. Thus, the Cu ISAS/NC catalyst offers high accessible surface area and large exposed active sites, facilitating the mass transport. According to the BET and ICP-AES analysis, the surface coverage of $\mathrm{Cu}$ atoms is estimated to be 0.0509 atoms $/ \mathrm{nm}^{2}$. The oxygen reduction reaction (ORR) performance of $\mathrm{NC}, \mathrm{Cu}$ ISAS/NC and commercial $\mathrm{Pt} / \mathrm{C}$ was evaluated by linear sweep voltammetry (LSV) technology in an $\mathrm{O}_{2}$-saturated $0.1 \mathrm{M} \mathrm{KOH}$ condition. As displayed in Fig. 2a, after $\mathrm{Cu}$ atoms doping, the $\mathrm{Cu}$ ISAS/NC catalyst provides the higher ORR activity with half-wave potential $\left(\mathrm{E}_{1 / 2}\right)$ of $(0.92 \mathrm{~V}$ vs RHE) (Fig. $2 \mathrm{~b})$, which is $52 \mathrm{mV}$ higher than that of $\mathrm{Pt} / \mathrm{C}$. This value is among the best ORR activity delivered by noble metal-free catalysts reported previously (Supplementary Table 2). At a potential of $0.9 \mathrm{~V}, \mathrm{Cu}$ ISAS/NC catalyst exhibits a much larger kinetic current density $\left(8.87 \mathrm{~mA} \mathrm{~cm}^{-2}\right)$ than those of $\mathrm{Pt} / \mathrm{C}\left(1.05 \mathrm{~mA} \mathrm{~cm}^{-2}\right)$ and $\mathrm{NC}\left(0.12 \mathrm{~mA} \mathrm{~cm}^{-2}\right)$. Additionally, the highest ORR kinetic process catalyzed by $\mathrm{Cu}$ ISAS/NC was further evidenced by a smaller Tafel slope of $59 \mathrm{mV} \mathrm{dec}^{-1}$ compared with that of $\mathrm{Pt} / \mathrm{C}\left(95 \mathrm{mV} \mathrm{dec}^{-1}\right)$ and $\mathrm{NC}$ matrix $\left(116 \mathrm{mV} \mathrm{dec}^{-1}\right)$ (Supplementary Fig. 14). The value of electron transfer number $(n)$ was calculated to be 3.99 (Supplementary Fig. 15), close to the theoretical value of 4.0 for oxygen reduction. The rotating ring-disk electrode (RRDE) tests further confirmed the four-electron pathway of $\mathrm{Cu}$ ISAS/NC catalyst in $\mathrm{KOH}$ (Supplementary Fig. 16). Additionally, the $\mathrm{Cu}$ ISAS/NC displays excellent stability after 20,000 cycles (Supplementary Fig. 17). This excellent robustness is ascribed to the highly stable atomic reactive sites, whose atomic dispersion remain after the durability (Supplementary Fig. 18). The Cu ISAS/NC could easily be scaled up to higher yields to satisfy the demand of large-scale production (Supplementary Fig. 19). To validate the implementation, the Cu ISAS/NC material was employed into a primary $\mathrm{Zn}$-air battery (Supplementary Fig. 20). As exhibited in Fig. 2c, the Zn-air battery using $\mathrm{Cu}$ ISAS/NC catalyst displays high performance with the maximum power density of up to $280 \mathrm{~mW} \mathrm{~cm}^{-2}$, superior to $\mathrm{Pt} / \mathrm{C}$-based Zn-air battery $\left(200 \mathrm{~mW} \mathrm{~cm}^{-2}\right)$, as well as other reported catalysts (Supplementary Table 3). At the discharge of $50 \mathrm{~mA} \mathrm{~cm}^{-2}$ (Fig. 2d), the specific capacity of the $\mathrm{Zn}$-air battery using the $\mathrm{Cu}$ ISAS/NC as air-cathode was estimated to be $\sim 736 \mathrm{mAh} \mathrm{g}^{-1}$. More importantly, the $\mathrm{Cu}$ ISAS/NC-based battery can robustly serve over $45 \mathrm{~h}$ with only a negligible drop of discharge voltage (Supplementary Fig. 21), indicating the excellent stability of the $\mathrm{Cu}$ ISAS/NC catalyst in practical Zn-air device.

DFT calculations. We further executed density functional theory (DFT) calculations to explore the nature of the active sites of the $\mathrm{Cu}$ ISAS/NC ORR catalyst. Based on the EXAFS analysis for the $\mathrm{Cu}$ ISAS/NC, we proposed two models containing $\mathrm{Cu}-\mathrm{N}_{3}$ structure, as shown in Fig. 3a, b, where the $\mathrm{Cu}$ in the $\mathrm{Cu}$ ISAS/NC is coordinated by three $\mathrm{N}$ atoms $\left(\mathrm{Cu}-\mathrm{N}_{3}\right)$ as well as one $\mathrm{C}$ atom and three $\mathrm{N}$ atoms $\left(\mathrm{Cu}-\mathrm{N}_{3}-\mathrm{C}\right)$, respectively. Considering that the carbon defect may be induced during the reduction of $\mathrm{Cu}-\mathrm{O}$ species, a $\mathrm{Cu}-\mathrm{N}_{3}$ model with a vacancy $\left(\mathrm{Cu}-\mathrm{N}_{3}-\mathrm{V}\right)$ was also constructed (Fig. 3c). According to the associative mechanism in alkaline medium and the correspondingly optimized configurations of the intermediates (Supplementary Fig. 22-24) as well as the free energies for each step (Supplementary Table 4) for the 

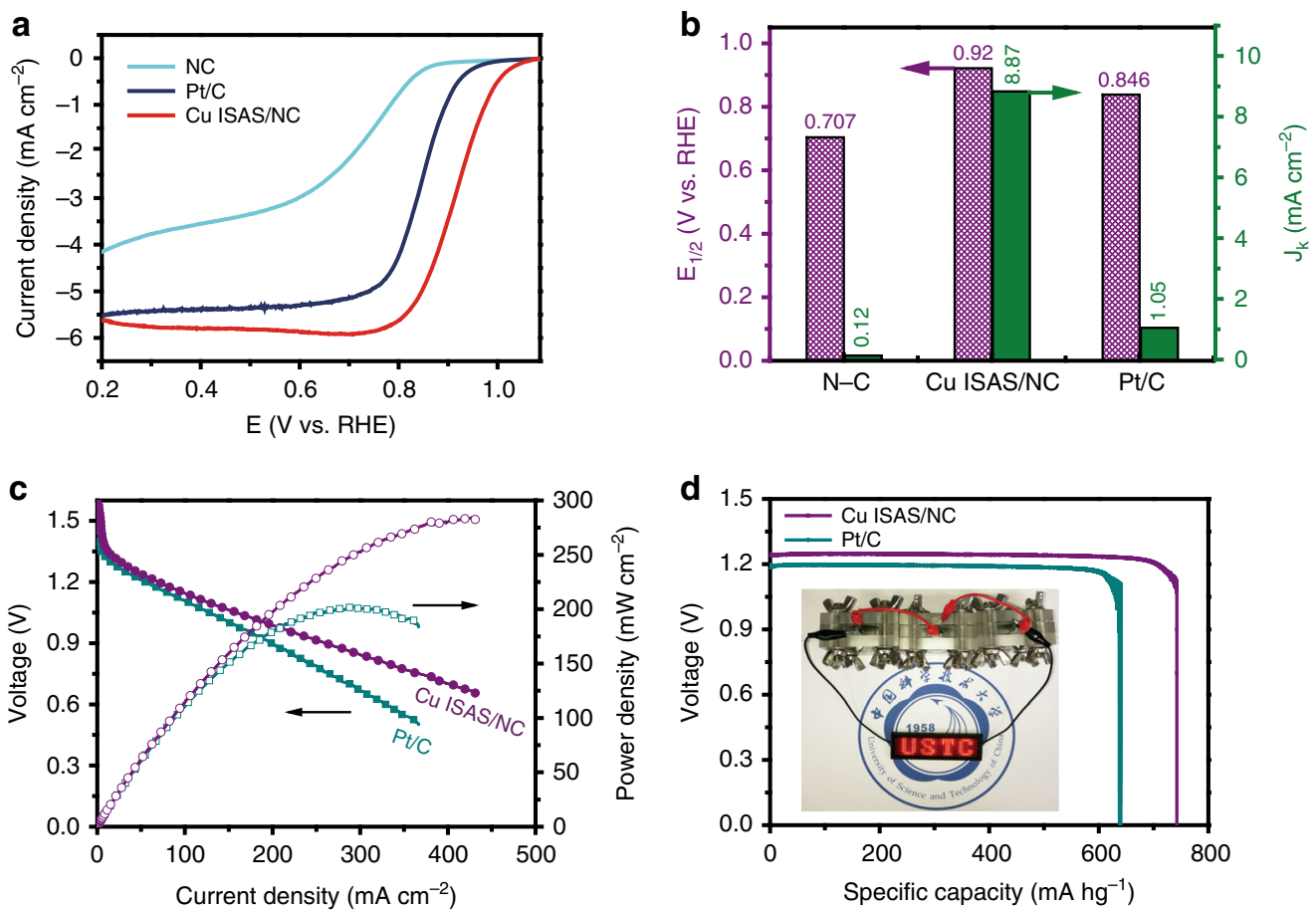

Fig. 2 Electrocatalytic oxygen reduction and Zn-air battery performances. a Linear sweep voltammetry (LSV) curves of NC, Cu ISAS/NC and Pt/C catalysts in $0.1 \mathrm{M} \mathrm{KOH}$ solution with a sweep rate of $10 \mathrm{mV} \mathrm{s}^{-1}$ and rotation rate of $1600 \mathrm{rpm}$. $\mathbf{b}$ half-wave potential $\left(E_{1 / 2}\right)$ and kinetic current density $\left(J_{\mathrm{K}}\right)$ of different catalysts. c Polarization and corresponding power density plots of Cu ISAS/NC and Pt/C-based Zn-air batteries. $\mathbf{d}$ The specific capacity of Cu ISAS/NC and $\mathrm{Pt} / \mathrm{C}$-based $\mathrm{Zn}$-air batteries at $50 \mathrm{~mA} \mathrm{~cm}^{-2}$. (Inset: a photograph showing light-emitting diode panel powered by three Cu ISAS/NC-based Zn-air batteries)

a

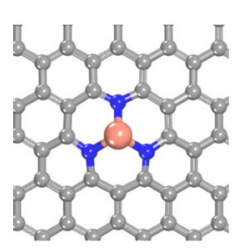

d

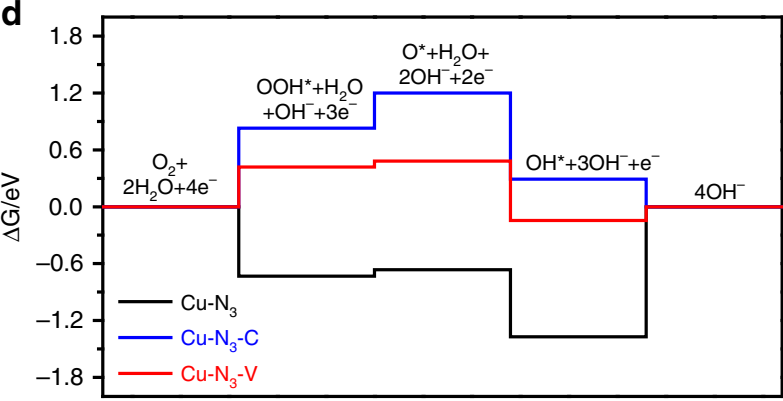

b
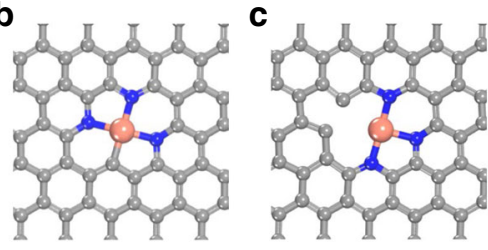

Reaction coordinate

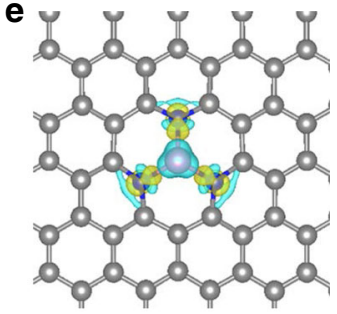

$\mathbf{f}$

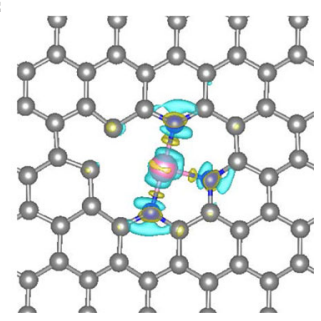

Fig. 3 DFT calculation. Top view of the optimized structures of the models of $\mathbf{a} C u-\mathrm{N}_{3}, \mathbf{b} \mathrm{Cu}-\mathrm{N}_{3}-\mathrm{C}$ and $\mathbf{c} \mathrm{Cu}-\mathrm{N}_{3}-\mathrm{V}$. Gray, blue and orange represent $\mathrm{C}$, N, and $\mathrm{Cu}$ atoms, respectively. $\mathbf{d}$ Free energy diagram for ORR process on these three models at the equilibrium potential $(U=0.40 \mathrm{~V}$ vs. NHE or $U=1.23 \mathrm{~V}$ vs $\mathrm{RHE}$ ) at $\mathrm{pH}=14$. The corresponding charge density difference of e $\mathrm{Cu}-\mathrm{N}_{3}$ and $\mathbf{f} \mathrm{Cu}-\mathrm{N}_{3}-\mathrm{V}$, in which light blue and yellow isosurfaces denote a decrease and increase of $0.005 \mathrm{e} / \AA^{3}$ for electronic density, respectively

ORR, the free energy diagrams of ORR processes were subsequently obtained at the equilibrium potential $(\mathrm{U}=0.40 \mathrm{~V}$ vs NHE at the $\mathrm{pH}=14$ ) over the single $\mathrm{Cu}$ active sites of these models and the results are shown in Fig. $3 \mathrm{~d}$. Clearly, for the $\mathrm{Cu}-\mathrm{N}_{3}$ model, the $\mathrm{OH}^{*}$ removal is the rate-determining step, while for the $\mathrm{Cu}-\mathrm{N}_{3}-\mathrm{C}$ and $\mathrm{Cu}-\mathrm{N}_{3}-\mathrm{V}$ models, the $\mathrm{OOH}^{*}$ formation is the ratedetermining step. Furthermore, the theoretical ORR overpotentials of these three models, an important measure of the
ORR catalyst performance ${ }^{27}$, were obtained from the correlated free energy profiles (Supplementary Fig. 25). Obviously, both the $\mathrm{Cu}-\mathrm{N}_{3}$ and the $\mathrm{Cu}-\mathrm{N}_{3}-\mathrm{C}$ models possess high theoretical ORR overpotentials $(1.37 \mathrm{eV}$ and $0.83 \mathrm{eV})$, which are much higher than the overpotential of the $\mathrm{Cu}$ ISAS/NC catalyst in our experiment. Interestingly, the theoretical overpotential of the $\mathrm{Cu}-\mathrm{N}_{3}-\mathrm{V}$ model, which is derived from introducing defect in $\mathrm{Cu}-\mathrm{N}_{3}-\mathrm{C}$ model, decreased darmatically compared to that of the $\mathrm{Cu}-\mathrm{N}_{3}$ and 
$\mathrm{Cu}-\mathrm{N}_{3}-\mathrm{C}$ models, indicating the important role of defect in the $\mathrm{Cu}-\mathrm{N}-\mathrm{C}$ ORR catalysts. Notably, even the defect is trapped by the adsorbed oxygen $\left(\mathrm{O}^{\star}\right)$ during the ORR process (Supplementary Fig. 26), the $\mathrm{Cu}-\mathrm{N}_{3}-\mathrm{V}$ model with $\mathrm{O}^{*}$ pre-adsorbed on the vacancy still show relatively low theoretical overpotential $(0.517 \mathrm{~V})$, indicating the $\mathrm{Cu}-\mathrm{N} 3-\mathrm{V}$ model as the possible active site of the $\mathrm{Cu}$ ISAS/NC ORR catalyst. The charge density difference shown in Fig. 3e, $\mathrm{f}$ indicate that the introduction of the defect will cause the inhomogeneous charge distribution around the $\mathrm{Cu}$ SAs. Such charge density asymmetry will lead to the synergistic effect of the defect and the $\mathrm{N}$ coordination around the $\mathrm{Cu}$ SAs and thus enhance the ORR activity ${ }^{19,28}$.

Interestingly, when the NC trapping agent was substituted by $\mathrm{N}$-doped reduced graphene oxide (N-rGO) and $\mathrm{N}$-doped carbon nanotubes (N-CNTs), we also successfully obtained isolated $\mathrm{Cu}$ ISAS/N-rGO (Fig. 4a, Supplementary Fig. 27 and Fig. 28) and $\mathrm{Cu}$ ISAS/N-CNTs (Fig. 4b, Supplementary Fig. 29 and Fig. 30) catalysts. The ORR performance for the $\mathrm{Cu}$ ISAS/N-CNTs and $\mathrm{Cu}$ ISAS/N-rGO are shown in Fig. S31. To test the universality of the high-temperature gas-transport route, we used different metal oxide powders (metal $=\mathrm{Mo}, \mathrm{Sn}$ ) to fabricate isolated M ISAS/NC materials. The AC HAADF-STEM images distinctly present isolated bright dots in Mo ISAS/NC (Fig. 4c and Supplementary Fig. 32) and Sn ISAS/NC (Fig. 4d and Supplementary Fig. 33), validating the atomically dispersed Mo and $\mathrm{Sn}$ atoms anchored on $\mathrm{N}$-doped carbons.

\section{Discussion}

We have demonstrated a versatile and universal gas-transport route to directly transform monolithic metal oxides into isolated single-atoms electrocatalysts. Under high temperature, the surface metal oxides were initially evaporated to generate volatile species, which were trapped and reduced by the N-rich carbon supports, forming the isolated $\mathrm{M}$ ISAS/NC catalysts $(\mathrm{M}=\mathrm{Cu}, \mathrm{Mo}, \mathrm{Sn})$. Impressively, the as-prepared $\mathrm{Cu}$ ISAS/NC catalyst has been demonstrated to endow excellent catalytic activity for ORR in an alkaline medium and achieve high performance for a $\mathrm{Zn}$-air battery. This work paves a way to directly prepare single atoms from bulk metal oxides.
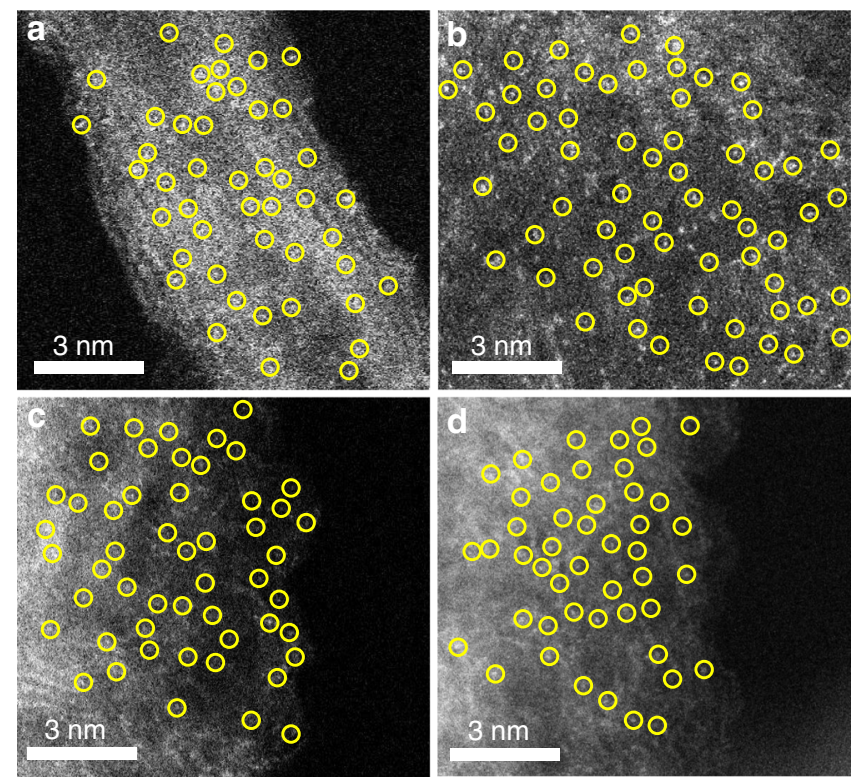

Fig. 4 Atomic structural characterizations AC HAADF-STEM images of a Cu ISAS/N-CNTs, b Cu ISAS/N-rGO, c Mo ISAS/NC, and d Sn ISAS/NC catalysts

\section{Methods}

Chemicals. Copper oxide $\left(\mathrm{Cu}_{2} \mathrm{O}\right)$, Molybdenum trioxide $\left(\mathrm{MoO}_{3}\right)$, tin dioxide $\left(\mathrm{SnO}_{2}\right)$ melamine $(\mathrm{M})$ were obtained from Sinopharm Chemical Reagents, China. Multi-walled carbon nanotubes (CNTs) was purchased from Alfa Aesar. Graphite powder (400 mesh) was obtained from XFNANO. Toray Carbon Paper (Toray TGP-H $\mathrm{H}^{-}$060, Toray Industries Inc.) was ultrasonically cleaned in ethanol. Analytical grade methanol $(\mathrm{CH} 3 \mathrm{OH})$, ethanol, zinc nitrate hexahydrate $(\mathrm{Zn}$

$\left.\left(\mathrm{NO}_{3}\right)_{2} \cdot 6 \mathrm{H}_{2} \mathrm{O}\right)$, 2-methylimidazole were purchased from Aldrich. All the chemicals were analytical grade and used without further purification.

Synthesis of NC. In a typical procedure, $3 \mathrm{~g} \mathrm{Zn}\left(\mathrm{NO}_{3}\right)_{2} \bullet 6 \mathrm{H}_{2} \mathrm{O}$ was dissolved in $50 \mathrm{ml}$ methanol and subsequently added into $100 \mathrm{ml}$ methanol containing $6.5 \mathrm{~g} \mathrm{2-}$ methylimidazole (MeIM) under vigorous stirring for $24 \mathrm{~h}$ at room temperature The as-obtained precipitates were centrifuged and washed with methanol three times and dried in vacuum at $60^{\circ} \mathrm{C}$ for overnight. The dried sample was placed in the porcelain boat. Then, the boat was heated at $950^{\circ} \mathrm{C}$ under $\mathrm{N}_{2}$ for $1 \mathrm{~h}$ with the heating rate of $5^{\circ} \mathrm{C} / \mathrm{min}$. After the temperature was down to room temperature, the mixture was stored in the glass bottle for further use.

Synthesis of $\mathbf{C u}$ ISAS/NC. In a normal procedure, the $\mathrm{Cu}_{2} \mathrm{O}$ power and the powder of NC $(100 \mathrm{mg})$ were separately placed on the porcelain boat. The porcelain boat was placed in a tube furnace and heated to $1000^{\circ} \mathrm{C}$ (heating rate $\left.5{ }^{\circ} \mathrm{C} / \mathrm{min}\right)$ in a stream of $\mathrm{N}_{2}(10 \mathrm{ml} / \mathrm{min})$ for $5 \mathrm{~h}$ to yield $\mathrm{Cu}$ ISAS/NC.

Synthesis of Cu ISAS/N-CNTs and Cu ISAS/N-rGO. The nitrogen-doped CNTs $(\mathrm{N}-\mathrm{CNTs})$ and nitrogen-doped reduced graphene oxide (N-rGO) were prepared by pyrolysis the mixtures of CNTs and $\mathrm{M}$ (mass ration: 1:2) and GO and $\mathrm{M}$ (mass ration: $1: 2$ ), respectively, at $1000^{\circ} \mathrm{C}$ for $1 \mathrm{~h}$ in flowing $\mathrm{N}_{2}$. Synthesis of Cu ISAS/N$\mathrm{CNTs}$ and $\mathrm{Cu}$ ISAS/N-rGO were the same as Cu ISAS/NC except using N-CNTs or $\mathrm{N}-\mathrm{rGO}$ as support.

Synthesis of Mo SAs/NC and Sn SAs/NC. The preparation of Mo ISAS/NC and $\mathrm{Sn}$ ISAS/NC is similar as $\mathrm{Cu}$ ISAS/NC except using $\mathrm{MoO}_{3}$ and $\mathrm{SnO}_{2}$ power. The temperature was changed as $600^{\circ} \mathrm{C}$ for Mo ISAS/NC.

Electrochemical measurements. All electrochemical tests were performed on $\mathrm{CHI}$ $760 \mathrm{E}$ electrochemical workstation with a three-electrode electrochemical cell, in which a graphite rod used as counter electrode, an $\mathrm{Ag} / \mathrm{AgCl}$ as reference electrode. The catalyst ink was prepared by ultrasonically $5 \mathrm{mg} \mathrm{Cu}$ ISAS/NC catalyst powder dispersed into $1 \mathrm{~mL}$ ethanol including $10 \mu \mathrm{L}$ 5\% Nafion (D520, Dupont Inc., USA). A volume of $10 \mu \mathrm{L}$ of the homogeneous catalyst ink was droped on a glassy carbon (GC) electrode with a diameter of $5 \mathrm{~cm}$. A flow of $\mathrm{O}_{2}$ was kept over the electrolyte during the tests so as to guarantee $\mathrm{O}_{2}$ saturation. LSV curves of the catalysts were conducted using a rotating disk electrode (RDE) with a scan rate of $10 \mathrm{mV} \mathrm{s}^{-1}$ at a rotating speed of $1600 \mathrm{rpm}$. The overall number of electrons transferred (n) during in oxygen reduction was calculated by Koutecky-Levich equation:

$$
\frac{1}{J}=\frac{1}{J_{\mathrm{L}}}+\frac{1}{J_{\mathrm{K}}}=\frac{1}{B \omega^{1 / 2}}+\frac{1}{J_{\mathrm{K}}}
$$

where $J$ is the measured current density, $J_{\mathrm{K}}$ and $J_{\mathrm{L}}$ are the kinetic and diffusionlimiting current densities, $\omega$ is the angular velocity $(\omega=2 \pi \mathrm{N}, N$ is the rotation speed), $B$ is Levich slope which is calculated as below:

$$
\mathrm{B}=0.62 n F C_{0} D_{0}^{2 / 3} V^{-1 / 6}
$$

where $\mathrm{F}$ is the Faraday constant $\left(96485 \mathrm{C} \mathrm{mol}^{-1}\right), C_{0}$ is the bulk concentration of $\mathrm{O}_{2}$ $\left(\left(1.2 \times 10^{-6} \mathrm{~mol} \mathrm{~cm}^{-3}\right), D_{0}\right.$ is the diffusion coefficient of $\mathrm{O}_{2}$ in $0.1 \mathrm{M} \mathrm{KOH}(1.9 \times$ $\left.10^{-5} \mathrm{~cm}^{2} \mathrm{~s}^{-1}\right)$, and $v$ is the kinematic viscosity of the electrolyte $\left(0.01 \mathrm{~cm}^{2} \mathrm{~s}^{-1}\right)$. Rotating ring-disk electrode (RRDE) measurements of the samples was measured to study the four-electron selectivity. The Pt ring electrode was biased at $1.2 \mathrm{~V}$ vs. RHE. The $\mathrm{H}_{2} \mathrm{O}_{2}$ yield and $\mathrm{n}$ per oxygen molecule were calculated by the following equations:

$$
\begin{gathered}
\% \mathrm{H}_{2} \mathrm{O}_{2}=200 \frac{I_{\mathrm{R}} / N}{I_{\mathrm{D}}+I_{\mathrm{R}} / N} \\
n=4 \frac{I_{\mathrm{D}}}{I_{\mathrm{D}}+I_{\mathrm{R}} / N}
\end{gathered}
$$

where $I_{\mathrm{D}}$ and $I_{\mathrm{R}}$ are the disk and ring currents, respectively. $N$ is the ring current collection efficiency (37\%).

Zinc-air (Zn-air) battery measurements. Primary Zn-air batteries tests were carried out in a home-built device. The air electrode was prepared by uniformly brushing the as-prepared catalyst ink onto carbon fiber paper $\left(1.0 \mathrm{~cm}^{2}\right)$ at $60^{\circ} \mathrm{C}$. All the catalyst loading of the air cathode with the $\mathrm{Cu}$ ISAS/NC and $\mathrm{Pt} / \mathrm{C}$ catalysts was $1.0 \mathrm{mg} \mathrm{cm}^{-2}$ unless otherwise stated. The polished commercial $\mathrm{Zn}$ foil was used as anode. The electrolyte in home-built $\mathrm{Zn}$-air battery is $6 \mathrm{M} \mathrm{KOH}$ electrolyte saturated with $\mathrm{O}_{2}$. The discharge polarization curve and stabilitly measurements were measured using the as-made device with $\mathrm{CHI} 760 \mathrm{E}$ electrochemical workstation. 
Computational details. All DFT calculations were conducted employing the Vienna ab initio simulation package (VASP) ${ }^{29-32}$. We used the projector augmented wave (PAW) method to depict the interactions between ion cores and valence electrons ${ }^{33}$. Meanwhile, we used the GGA-PBE to describe the exchangecorrelation functional ${ }^{34,35}$. The solution of the Kohn-Sham equations was expanded in a plane wave basis set with a cutoff energy of $400 \mathrm{eV}$. The Brillouin zone sampling was performed using a Monkhorst-Pack grid ${ }^{36}$, and electronic occupancies were determined in light of a Gaussian smearing with a width of $0.05 \mathrm{eV}$. Considering that the solvent may influence the calculation results, we employed VASPsol to include the implicit solvation effect in the calculation ${ }^{37}$. A force-based conjugated gradient method was used to optimize the geometries in all the calculations $^{38}$. Saddle points and minima were converged when the maximum force in each degree of freedom was less than $0.03 \mathrm{eV}^{-1}$. Bader charge analysis was implemented with a fast algorithm developed by Henkelman and coworkers, and the core charges were included in the partitions ${ }^{39,40}$.

All of the $\mathrm{Cu}$ ISAS supported on the graphene models are constructed based on the graphene basal plane model with the supercell of $5 \times 3 \sqrt{3} \times 1(12.30 \AA \times$ $12.78 \AA \times 15.00 \AA$ ). The Monkhorst-Pack meshes of $3 \times 3 \times 1$ k-point samplings in the surface Brillouin zones were used for these models. All models constructed are shown in Supplementary Fig. 21. The ORR were further analyzed on the $\mathrm{Cu}$ sites of these models. The ORR can proceed through a two-electron pathway in which $\mathrm{O}_{2}$ is reduced to $\mathrm{H}_{2} \mathrm{O}_{2}$, or a four-electron process that completely reduces $\mathrm{O}_{2}$ to $\mathrm{H}_{2} \mathrm{O}$. Here, we calculate the complete reduction cycle based on the experimental observations that the four-electron was the dominant mechanism on the $\mathrm{Cu}$ ISAS/NC catalyst. In alkaline condition, the ORR step can be summarized as follows:

$$
\begin{gathered}
\mathrm{O}_{2}^{*}+2 \mathrm{H}_{2} \mathrm{O}+4 e^{-} \rightarrow \mathrm{OOH}^{*}+\mathrm{OH}^{-} \mathrm{H}_{2} \mathrm{O}+3 e^{-} \\
\mathrm{OOH}^{*}+\mathrm{OH}^{-}+\mathrm{H}_{2} \mathrm{O}+3 e^{-} \rightarrow 2 \mathrm{OH}^{-}+\mathrm{O}^{*}+\mathrm{H}_{2} \mathrm{O}+2 e^{-} \\
2 \mathrm{OH}^{-}+\mathrm{O}^{*}+\mathrm{H}_{2} \mathrm{O}+2 e^{-} \rightarrow 3 \mathrm{OH}^{-}+\mathrm{OH}^{*}+e^{-} \\
3 \mathrm{OH}^{-}+\mathrm{OH}^{*}+e^{-} \rightarrow 4 \mathrm{OH}^{-} \\
\text {Overall }: \mathrm{O}_{2}+2 \mathrm{H}_{2} \mathrm{O}+4 e^{-} \rightarrow 4 \mathrm{OH}^{-}
\end{gathered}
$$

In ORR, the rate-determining steps were reported as the adsorption of $\mathrm{O}_{2}$ as $\mathrm{OOH}^{*}(5)$ or the desorption of $\mathrm{OH}^{*}(7)^{41}$ and at all the steps would influence the corresponding ORR activity. Here, we used these reactions to derive the ORR thermochemistry. The free energy diagrams for ORR were determined according to the method proposed by Nørskov et al. ${ }^{27}$. We drew the free energy diagrams by setting up the reference electrode as the NHE. It is noted that the equilibrium potential $\mathrm{U}^{0}$ for ORR at $\mathrm{pH}=14$ was determined to be $0.402 \mathrm{~V}$ vs NHE, where the reactant and product are at the same energy level ${ }^{42}$. In our calculations, the ORR was analyzed using intermediate species associated with one electron transfer at a time. The free energy change from initial states to final states of the reaction is calculated as follows:

$$
\Delta \mathrm{G}=\Delta E+\Delta \mathrm{ZPE}-T \Delta S+\Delta G_{\mathrm{U}}+\Delta G_{\mathrm{pH}}
$$

where $\Delta \mathrm{E}$ is the reaction energy of reactants and products adsorbed on catalyst surface, $\Delta \mathrm{ZPE}$ is the change of zero-point energy, $\Delta G_{\mathrm{U}}=-\mathrm{eU}$ ( $U$ is the electrode potential and $e$ is the transferred charge), $T$ is the temperature, $\Delta S$ is the entropy change at $298.15 \mathrm{~K}, \Delta G_{\mathrm{pH}}$ is the correction of the $\mathrm{H}^{+}$free energy, which is calculated as $\Delta G_{\mathrm{pH}}=\mathrm{k}_{\mathrm{B}} \mathrm{T} \times \ln 10 \mathrm{pH}$, where $k_{\mathrm{B}}$ is the Boltzmann constant. The free energy of $\mathrm{H}_{2}, \mathrm{H}_{2} \mathrm{O}$, and $\mathrm{O}_{2}$ was the same as previous literature reported ${ }^{27}$. The free energy of $\left(\mathrm{H}^{+}+\mathrm{e}^{-}\right)$in solution at standard conditions of $U=0$ and $\mathrm{pH}=0$ is equal to that of $1 / 2 \mathrm{H}_{2}$ according to a computational hydrogen electrode model suggested by Nørskov et al. ${ }^{27}$. The free energy of $\mathrm{OH}^{-}$was calculated from the reaction $\mathrm{H}^{+}+\mathrm{OH}^{-} \rightarrow \mathrm{H}_{2} \mathrm{O}$, which is in equilibrium in water solution ${ }^{43,44}$. The entropies and vibrational frequencies of the species in gas phase were taken from the previous literatures ${ }^{27}$. Zero-point energy and entropies of the adsorbed species were calculated from the vibrational frequencies. To acquire the reaction free energy of ORR process, we firstly calculated the adsorption free energy of $\mathrm{O}^{*}, \mathrm{OH}^{*}$, and $\mathrm{OOH}^{*}$. Due to the difficulies in obtaining the exact free energy of $\mathrm{OOH}, \mathrm{O}$, and $\mathrm{OH}$ radicals in the electrolyte solution, the adsorption free energy of these intermediates are all calculated based on the relative free energy of stoichiometrically appropriate amounts of $\mathrm{H}_{2} \mathrm{O}(\mathrm{g})$ and $\mathrm{H}_{2}(\mathrm{~g})$ according to the previous studies ${ }^{27}$. The corresponding reaction free energies of equations 5 to $8\left(\Delta \mathrm{G}_{1}\right.$ to $\left.\Delta \mathrm{G}_{4}\right)$ are obtained based on the adsorption free energy of $\mathrm{OOH}, \mathrm{O}$, and $\mathrm{OH}$ and the results are shown in Supplementary Table 4 . The overpotential $\eta_{\text {ORR }}$ of the whole ORR process can be calculated at the equilibrium potential $(U=0.40 \mathrm{~V}$ vs. $\mathrm{NHE}$ at $\mathrm{pH}=14$ ) by the following equations:

$$
\eta_{\mathrm{ORR}}=\max \left\{\Delta G_{1} ; \Delta G_{2} ; \Delta G_{3} ; \Delta G_{4}\right\} / e
$$

\section{Data availability}

The data that support the findings of this study are available from the corresponding author upon request.
Received: 29 November 2018 Accepted: 31 July 2019

Published online: 19 August 2019

\section{References}

1. Yang, X.-F. et al. Single-atom catalysts: a new Frontier in heterogeneous catalysis. Acc. Chem. Res. 46, 1740-1748 (2013).

2. Chen, Z. et al. A heterogeneous single-atom palladium catalyst surpassing homogeneous systems for suzuki coupling. Nat. Nanotechnol. 13, 702-707 (2018).

3. Zhu, C., Fu, S., Shi, Q., Du, D. \& Lin, Y. Single-atom electrocatalysts. Angew. Chem. Int. Ed. 56, 13944-13960 (2017).

4. Yan, H. et al. Single-atom Pd1/graphene catalyst achieved by atomic layer deposition: remarkable performance in selective hydrogenation of 1, 3butadiene. J. Am. Chem. Soc. 137, 10484-10487 (2015).

5. Cheng, N. et al. Platinum single-atom and cluster catalysis of the hydrogen evolution reaction. Nat. Commun. 7, 13638 (2016).

6. Kaden, W. E., Wu, T., Kunkel, W. A. \& Anderson, S. L. Electronic structure controls reactivity of size-selected Pd clusters adsorbed on $\mathrm{TiO}_{2}$ surfaces. Science 326, 826-829 (2009).

7. Ding, K. et al. Identification of active sites in CO oxidation and water-gas shift over supported Pt catalysts. Science 350, 189-192 (2015).

8. Zheng, Y. et al. Molecule-level g- $\mathrm{C}_{3} \mathrm{~N}_{4}$ coordinated transition metals as a new class of electrocatalysts for oxygen electrode reactions. J. Am. Chem. Soc. 139, 3336-3339 (2017).

9. Qiao, B. et al. Single-atom catalysis of $\mathrm{CO}$ oxidation using $\mathrm{Pt}_{1} / \mathrm{FeOx}$. Nat. Chem. 3, 634-641 (2011).

10. Liu, P. et al. Photochemical route for synthesizing atomically dispersed palladium catalysts. Science 352, 797-800 (2016).

11. Kwon, Y., Kim, T. Y., Kwon, G., Yi, J. \& Lee, H. Selective activation of methane on single-atom catalyst of rhodium dispersed on zirconia for direct conversion. J. Am. Chem. Soc. 139, 17694-17699 (2017).

12. Nie, L. et al. Activation of surface lattice oxygen in single-atom $\mathrm{Pt} / \mathrm{CeO}_{2}$ for low-temperature CO oxidation. Science 358, 1419-1423 (2017).

13. Wei, S. et al. Direct observation of noble metal nanoparticles transforming to thermally stable single atoms. Nat. Nanotechnol. 13, 856-861 (2018).

14. Yang, J. et al. In situ thermal atomization to convert supported nickel nanoparticles into surface-bound nickel single-atom catalysts. Angew. Chem. Int. Ed. 57, 14095-14100 (2018).

15. Qu, Y. et al. Direct transformation of bulk copper into copper single sites via emitting and trapping of atoms. Nat. Catal. 1, 781-786 (2018).

16. Zhu, C., Wen, Y., van Aken, P. A., Maier, J. \& Yu, Y. High lithium storage performance of $\mathrm{FeS}$ nanodots in porous graphitic carbon nanowires. $A d v$. Funct. Mater. 25, 2335-2342 (2015).

17. Li, J.-S. et al. Nitrogen-doped Fe/Fe ${ }_{3} \mathrm{C} @$ graphitic layer/carbon nanotube hybrids derived from MOFs: efficient bifunctional electrocatalysts for ORR and OER. Chem. Commun. 51, 2710-2713 (2015).

18. Yang, Z. K. et al. Synthesis of nanoporous structured iron carbide/ $\mathrm{Fe}-\mathrm{N}$-carbon composites for efficient oxygen reduction reaction in $\mathrm{Zn}$-air batteries. J. Mater. Chem. A 4, 19037-19044 (2016).

19. Qin, Y. et al. Aluminum and Nitrogen codoped Graphene: Highly active and durable electrocatalyst for oxygen reduction reaction. ACS Catal. 9, 610-619 (2019).

20. Wang, J. et al. Design of N-coordinated dual-metal sites: a stable and active Ptfree catalyst for acidic oxygen reduction reaction. J. Am. Chem. Soc. 139, 17281-17284 (2017).

21. Wang, S. et al. Free-standing nitrogen-doped carbon nanofiber films: integrated electrodes for sodium-ion batteries with ultralong cycle life and superior rate capability. Adv. Energy Mater. 6, 1502217 (2016).

22. $\mathrm{Wu}, \mathrm{H}$. et al. Highly doped and exposed $\mathrm{Cu}(\mathrm{i})-\mathrm{N}$ active sites within graphene towards efficient oxygen reduction for zinc-air batteries. Energy Environ. Sci. 9, 3736-3745 (2016).

23. Mane, G. P. et al. Highly ordered nitrogen-rich mesoporous carbon nitrides and their superior performance for sensing and photocatalytic hydrogen generation. Angew. Chem. Int. Ed. 56, 8481-8485 (2017).

24. Yang, H. B. et al. Identification of catalytic sites for oxygen reduction and oxygen evolution in $\mathrm{N}$-doped graphene materials: Development of highly efficient metal-free bifunctional electrocatalyst. Sci. Adv. 2, e1501122 (2016).

25. Ehlert, C., Unger, W. E. \& Saalfrank, P. C K-edge NEXAFS spectra of graphene with physical and chemical defects: a study based on density functional theory. Phys. Chem. Chem. Phys. 16, 14083-14095 (2014).

26. Qu, Y. et al. Thermal emitting strategy to synthesize atomically dispersed $\mathrm{Pt}$ metal sites from bulk Pt metal. J. Am. Chem. Soc. 141, 4505-4509 (2019).

27. Nørskov, J. K. et al. Origin of the overpotential for oxygen reduction at a fuelcell cathode. J. Phys. Chem. B 108, 17886-17892 (2004).

28. Lei, Z. C. et al. Nitrified coke wastewater sludge flocs: an attractive precursor for N, S dual-doped graphene-like carbon with ultrahigh capacitance and oxygen reduction performance. J. Mater. Chem. A 5, 2012-2020 (2017). 
29. Kresse, G. \& Hafner, J. Ab initio molecular dynamics for liquid metals. Phys. Rev. B 47, 558 (1993).

30. Kresse, G. \& Hafner, J. Ab initio molecular-dynamics simulation of the liquidmetal-amorphous-semiconductor transition in germanium. Phys. Rev. B 49, 14251 (1994).

31. Kresse, G. \& Furthmüller, J. Efficiency of ab-initio total energy calculations for metals and semiconductors using a plane-wave basis set. Comp. Mater. Sci. 6, 15-50 (1996)

32. Kresse, G. \& Furthmüller, J. Efficient iterative schemes for ab initio totalenergy calculations using a plane-wave basis set. Phys. Rev. B 54, 11169 (1996).

33. Blöchl, P. E. Projector augmented-wave method. Phys. Rev. B 50, 17953 (1994).

34. Kresse, G. \& Joubert, D. From ultrasoft pseudopotentials to the projector augmented-wave method. Phys. Rev. B 59, 1758 (1999).

35. Perdew, J. P., Burke, K. \& Ernzerhof, M. Generalized gradient approximation made simple. Phys. Rev. Lett. 77, 3865 (1996).

36. Monkhorst, H. J. \& Pack, J. D. Special points for Brillouin-zone integrations. Phys. Rev. B 13, 5188 (1976).

37. Mathew, K., Sundararaman, R., Letchworth-Weaver, K., Arias, T. A. \& Hennig, R. G. Implicit solvation model for density-functional study of nanocrystal surfaces and reaction pathways. J. Chem. Phys. 140, 084106 (2014).

38. Blöchl, P. E., Jepsen, O. \& Andersen, O. K. Improved tetrahedron method for Brillouin-zone integrations. Phys. Rev. B 49, 16223 (1994).

39. Henkelman, G., Arnaldsson, A. \& Jónsson, H. A fast and robust algorithm for Bader decomposition of charge density. Comp. Mater. Sci. 36, 354-360 (2006).

40. Sanville, E., Kenny, S. D., Smith, R. \& Henkelman, G. Improved gridbased algorithm for Bader charge allocation. J. Comput. Chem. 28, 899-908 (2007).

41. Li, M., Zhang, L., Xu, Q., Niu, J. \& Xia, Z. N-doped graphene as catalysts for oxygen reduction and oxygen evolution reactions: theoretical considerations. J. Catal. 314, 66-72 (2014).

42. Zhang, J., Zhao, Z., Xia, Z. \& Dai, L. A metal-free bifunctional electrocatalyst for oxygen reduction and oxygen evolution reactions. Nat. Nanotechnol. 10, 444 (2015).

43. Yu, L., Pan, X., Cao, X., Hu, P. \& Bao, X. Oxygen reduction reaction mechanism on nitrogen-doped graphene: a density functional theory study. J. Catal. 282, 183-190 (2011).

44. Dai, L., Xue, Y., Qu, L., Choi, H. \& Baek, J. Metal-free catalysts for oxygen reduction reaction[J]. Chem. Rev. 115, 4823-4892 (2015).

\section{Acknowledgements}

This work was supported by National Key R\&D Program of China 2017YFA (0208300) and (0700104), the National Natural Science Foundation of China (21522107, 21671180, and 21802132), China Postdoctoral Science Foundation (2018M632536) and Funda- mental Research Funds for the Central Universities (WK2060190093). We thank the photoemission endstations BL1W1B in Beijing Synchrotron Radiation Facility (BSRF), BL14W1 in Shanghai Synchrotron Radiation Facility (SSRF), BL10B, and BL11U in National Synchrotron Radiation Laboratory (NSRL) for the help in characterizations.

\section{Author contributions}

Z.Y. performed the sample synthesis, characterization, electrochemical measurements, and wrote the manuscript. B.C. and X.D. performed density functional theory (DFT) calculations. W.C. and Q.X. carried out the XAFS measurements and discussion. Q.Z. helped to the HAADF-STEM measurements of samples. Y.Q., F.Z., and C.Z helped to analyze the data modify the paper. Y.W. conceived the idea and wrote the paper. All authors contributed to the preparation of the manuscript.

\section{Additional information}

Supplementary Information accompanies this paper at https://doi.org/10.1038/s41467 019-11796-4.

Competing interests: The authors declare no competing interests.

Reprints and permission information is available online at http://npg.nature.com/ reprintsandpermissions/

Peer review information: Nature Communications thanks Guoxiong Wang, and the other, anonymous, reviewer(s) for their contribution to the peer review of this work

Publisher's note: Springer Nature remains neutral with regard to jurisdictional claims in published maps and institutional affiliations.

\section{(c) (1)}

Open Access This article is licensed under a Creative Commons Attribution 4.0 International License, which permits use, sharing, adaptation, distribution and reproduction in any medium or format, as long as you give appropriate credit to the original author(s) and the source, provide a link to the Creative Commons license, and indicate if changes were made. The images or other third party material in this article are included in the article's Creative Commons license, unless indicated otherwise in a credit line to the material. If material is not included in the article's Creative Commons license and your intended use is not permitted by statutory regulation or exceeds the permitted use, you will need to obtain permission directly from the copyright holder. To view a copy of this license, visit http://creativecommons.org/ licenses/by/4.0/.

(C) The Author(s) 2019 